\title{
Gaussian MIMO relay channel with orthogonal channel components
}

\author{
Zouhair Al-qudah
}

\begin{abstract}
The Gaussian multiple-input multiple-output (MIMO) orthogonal relay channel (ORC) is investigated. The transmission from source to relay is done over a channel that is orthogonal to source-destination and relay-destination channels. Practically, this assumption is made such that many communication devices from different technologies are exploited in relaying the source's signal into its destination. For this channel model, the capacity is initially derived. Thereafter, we propose a transmission algorithm to achieve the derived capacity. Further, to support our theoretical results, many numerical examples are presented.
\end{abstract}

K e y w o r d s: channel capacity, MIMO system, multiple antennas, multiple access channel, orthogonal frequency bands

\section{Introduction}

In this paper, we consider the relay channel in which the source-destination pair is augmented by a third node, the relay. For the sake of forwarding the source's signal into its destination, different relaying techniques may be used. These techniques include decode-and-forward (DF), amplify-and-forward (AF), and compress-and-forward $(\mathrm{CF})$. Further, in the case that the nodes are equipped with single antenna for transmission and reception, which is commonly known as single-input single-output (SISO), the authors in [1] derived the capacity in many different cases including the degraded relay channel and the general relay channel. In a step forward, the authors in [2] established the capacity of the relay channel in which the transmission from source to relay is performed over a channel that is orthogonal, in frequency, to sourcedestination and relay-destination channels. Further, this capacity result is shown to be equal to the max-flow min-cut upper bound of the general relay channel [2]. In other words, employing a relay that can receive and then transmit over orthogonal channels achieve the capacity of the relay channel. Further, transmission over orthogonal channels, in which different frequency bands are used for either transmission or reception, are studied in many different multi-users scenarios [3-5]. For instance, different frequency bands are exploited to transmit to the relay and the intended receiver [5]. Practically, the importance of transmission and/or reception over different frequency bands is to let many communication devices from various technologies to extend and improve the system performance.

For the sake of increasing the achievable capacity of a given communication system, Telatar [6] used multiple antennas for transmission and also a set of antennas for reception. To achieve the capacity, Telatar used waterfilling technique, which was proposed in [7], for transmis- sion in the case that channel state information (CSI) is available at the transmitter. Furthermore, in the case of no CSI at transmitter, Telatar showed that equal power can be assigned to all transmit antennas to achieve the capacity. Then, the multiple-antenna system was expanded to many multi users communication systems like MIMO broadcast channel [8], MIMO relay channel [9-12], and massive MIMO networks [13]. For example, the authors in [9] derived the capacity of the MIMO relay channel in the case that the relay can DF the source's signal. In addition, the case that the relay can AF was studied in [10].

In this paper, we consider the transmission over Gaussian MIMO-ORC in which all nodes are equipped with multiple antennas. In addition, the transmission from source to relay is operated over a channel that is orthogonal to source-destination and relay-destination channels. As explained before, this communication scenario may model a multi-user communication model with multiple standards. In particular, the transmission from source to destination may get advantage of the availability of a third node, the relay. This relay may operate over different frequency bands such that it can receive over a frequency band and the transmission is performed over another frequency band.

Specifically, in this paper, we first derive the achievable capacity of the MIMO-ORC. Precisely, the capacity of the SISO Gaussian orthogonal relay channel is extended and derived for the MIMO Gaussian orthogonal relay channel. As will be shown in the derivation, this extension is not straightforward. Thereafter, an iterative algorithm is developed to achieve the capacity. Then, many numerical examples are presented to show the value of employing multiple antennas for transmission and reception at the nodes forming the relay channel.

Notations: In this paper, we use $\dagger$ to denote for the conjugate transpose, $\mathbf{E}$ is used for referring to the expec-

\footnotetext{
1 Communication Engineering department at Al-Hussein bin Talal University, Ma'an, Jordan, qudahz@ahu.edu.jo
} 


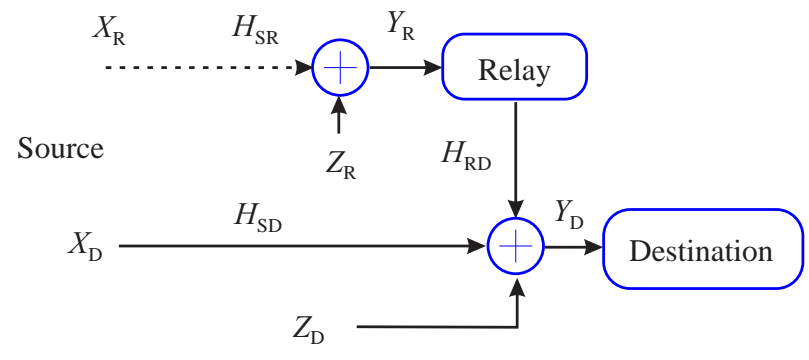

Fig. 1. Gaussian MIMO orthogonal relay channel. Different line formats denote different frequency bands.

tation operator, Cov is used to denote for the covariance, Var represents the variance, $I_{N}$ is used to exemplify an identity matrix of size $N$, and $\mathcal{C N}(\mu, \Sigma)$ is used to symbolize the distribution of a circularly symmetric complex Gaussian vector of mean $\mu$ and covariance matrix $\Sigma$.

\section{System model}

depicted in Fig. 1, the considered MIMO-ORC mainly consists of three nodes: source, destination, and an intermediate node, the relay. In this channel model, there are $\mathbf{M}_{S}$ transmit antennas at source, $\mathbf{N}_{D}$ receive antennas at destination, and the relay is equipped with $\mathbf{N}_{R}$ receive antennas and $\mathbf{M}_{R}$ transmit antennas. The Gaussian vector received signals at relay, $\mathbf{Y}_{R} \in \mathcal{C}^{N_{R} \times 1}$, and destination $\mathbf{Y}_{D} \in \mathcal{C}^{N_{D} \times 1}$, are respectively given by

$$
\begin{gathered}
\mathbf{Y}_{R}=\mathbf{H}_{S R} \mathbf{X}_{R}+\mathbf{Z}_{R} \\
\mathbf{Y}_{D}=\mathbf{H}_{S D} \mathbf{X}_{D}+\mathbf{H}_{R D} \mathbf{X}_{1}+\mathbf{Z}_{D}
\end{gathered}
$$

where

- $\mathbf{H}_{S R} \in \mathcal{C}^{N_{R} \times M_{S}}, \mathbf{H}_{S D} \in \mathcal{C}^{N_{D} \times M_{S}}$, and $\mathbf{H}_{R D} \in$ $\mathcal{C}^{N_{D} \times M_{R}}$ are the channel gains as shown in Fig. 1 . These channel gains are assumed to be constant and globally known.

- Over different frequency bands, the source transmits the signals $\mathbf{X}_{D} \in \mathcal{C}^{M_{S} \times 1}$ and $\mathbf{X}_{R} \in \mathcal{C}^{M_{S} \times 1}$ to both the destination and the relay, respectively. The average power $P_{S}$ at the transmitter is exploited to send both the signals $\mathbf{X}_{D}$ and $\mathbf{X}_{R}$ such that $\mathbf{E}\left[\mathbf{X}_{D}^{\dagger} \mathbf{X}_{D}\right] \leq \alpha P_{S}$ and $\mathbf{E}\left[\mathbf{X}_{R}^{\dagger} \mathbf{X}_{R}\right] \leq(1-\alpha) P_{S}=\bar{\alpha} P_{S} . \mathbf{X}_{1} \in \mathcal{C}^{M_{R} \times 1}$ is the transmitted signal from relay to destination with average power limited by $P_{R}$. Specifically, the relay initially decodes the received signal and then forwards $\mathbf{X}_{1 i}=\mathbf{F}\left(\mathbf{Y}_{R[i-1]}\right)$ which denotes that the signal $\mathbf{X}_{1 i}$, at time instant $i$, is a function of the signal $\mathbf{Y}_{R[i-1]}$. Further, the noise signals $\mathbf{Z}_{R} \in \mathcal{C}^{N_{R} \times 1}$ at relay and $\mathbf{Z}_{D} \in \mathcal{C}^{N_{D} \times 1}$ at destination are independent circularly symmetric additive Gaussian random variables with distributions $\mathcal{C N}\left(0, I_{N_{R}}\right)$ and $\mathcal{C N}\left(0, I_{N_{D}}\right)$, respectively.

\section{Capacity analysis}

As a matter of fact, the capacity is analyzed and consequently derived based on the following discussion.

Theorem 1. The capacity of the MIMO-ORC, $C$, is given by

$$
C=\min \left\{C_{1}, C_{2}+C_{3}\right\}
$$

where

$$
\begin{gathered}
C_{1}=\log \left[\operatorname { d e t } \left(I_{N_{D}}+\mathbf{H}_{S D} \Sigma_{D D} \mathbf{H}_{S D}^{\dagger}+\mathbf{H}_{R D} \Sigma_{1 D} \mathbf{H}_{S D}^{\dagger}+\right.\right. \\
\left.\left.+\mathbf{H}_{S D} \Sigma_{D 1} \mathbf{H}_{R D}^{\dagger}+\mathbf{H}_{R D} \Sigma_{11} \mathbf{H}_{R D}^{\dagger}\right)\right] \\
C_{2}=\log \left[\operatorname{det}\left(I_{N_{R}}+\mathbf{H}_{S R} \Sigma_{R R} \mathbf{H}_{S R}^{\dagger}\right)\right] \\
C_{3} \leq \log \left[\operatorname{det}\left(I_{N_{D}}+\left(1-\rho^{2}\right) \mathbf{H}_{S D} \Sigma_{D D} \mathbf{H}_{S D}^{\dagger}\right)\right]
\end{gathered}
$$

where $\rho$ represents the correlation coefficient, $\Sigma_{D D}, \Sigma_{11}$, $\Sigma_{R R}$, and $\Sigma_{1 D}$ are the covariance matrices. These terms are defined in the proof.

\section{Remark 1 .}

- To maximize the minimum achievable capacity, the average allocated power at the source and the relay are optimized such that the multiple access part, $\left(C_{1}\right)$, and the broadcast part, $\left(C_{2}+C_{3}\right)$, are almost equal.

- The source may use different number of antennas to transmit its signals. For example, the source may employ $M_{S D}$ antennas to send $\mathbf{X}_{D}$ and $M_{S R}$ antennas to transmit $\mathbf{X}_{R}$.

- Water-filling in conjunction with antenna selection can be used to significantly improve the achievable capacity [11]. In particular, using the water-filling algorithm can help to efficiently allocate the available average power toward using antennas with better conditions. This technique may reduce the number of transmit antennas at both the source and the relay. For instance, the source may use $M_{S D}^{*}$ to transmit to destination and $M_{S R}^{*}$ to send to relay.

We start the proof by introducing the following proposition.

Proposition 1 [2]. The capacity of the discrete memoryless MIMO-ORC with orthogonal channel components is expressed by

$C=\max \min$

$$
\left\{I\left(\mathbf{X}_{R} ; \mathbf{Y}_{R} \mid \mathbf{X}_{1}\right)+I\left(\mathbf{X}_{D} ; \mathbf{Y}_{D} \mid \mathbf{X}_{1}\right), I\left(\mathbf{X}_{D}, \mathbf{X}_{1}, \mathbf{Y}_{D}\right)\right\} .
$$

It is good to pay attention that this capacity is equal to the max-flow min-cut upper bound of the general MIMO relay channel [2]. We first derive the achievable rates of the Gaussian MIMO-ORC in the phase of broadcasting. Precisely, in this phase, the source transmits different signals to both the relay and the destination. Initially, the 
transmission rate from the source to the relay is given by the term $I\left(\mathbf{X}_{R} ; \mathbf{Y}_{R} \mid \mathbf{X}_{\mathbf{1}}\right)$ and then is obtained as follows

$$
\begin{aligned}
& C_{2}=I\left(\mathbf{X}_{R} ; \mathbf{Y}_{R} \mid \mathbf{X}_{1}\right)= \\
& =h\left(\mathbf{Y}_{R} \mid \mathbf{X}_{1}\right)-h\left(\mathbf{Y}_{R} \mid \mathbf{X}_{R}, \mathbf{X}_{1}\right)= \\
& =h\left(\mathbf{Y}_{R} \mid \mathbf{X}_{1}\right)-h\left(\mathbf{Y}_{R} \mid \mathbf{X}_{R}\right) \stackrel{\left(a_{1}\right)}{\leq} h\left(\mathbf{Y}_{R}\right)-h\left(\mathbf{Y}_{R} \mid \mathbf{X}_{R}\right)= \\
& =h\left(\mathbf{Y}_{R}\right)-h\left(\mathbf{Z}_{R}\right)=h\left(\mathbf{Y}_{R}\right)-\log \left[(\pi e)^{N_{D}} \operatorname{det}\left(I_{N_{R}}\right)\right] \\
& \stackrel{\left(a_{2}\right)}{=} \max _{\Sigma_{R R}} \log \left[\operatorname{det}\left(I_{N_{R}}+\mathbf{H}_{S R} \Sigma_{R R} \mathbf{H}_{S R}^{\dagger}\right)\right],
\end{aligned}
$$

where step $\left(a_{1}\right)$ follows since $\mathbf{Y}_{\mathbf{R}}$ is independent of $\mathbf{X}_{\mathbf{1}}$, and step $\left(a_{2}\right)$ follows again since circularly symmetric complex Gaussian distribution maximizes the entropy [6]. Further, the correlation matrix $\Sigma_{R R}$ is obtained by evaluating $E\left[\mathbf{X}_{R} \mathbf{X}_{R}^{\dagger}\right]$.

Then, the achievable rate from the source to the destination can be described by the part $I\left(\mathbf{X}_{D} ; \mathbf{Y}_{D} \mid \mathbf{X}_{1}\right)$ and is derived as follows

$$
\begin{aligned}
& C_{3}=I\left(\mathbf{X}_{D} ; \mathbf{Y}_{D} \mid \mathbf{X}_{1}\right)=h\left(\mathbf{Y}_{D} \mid \mathbf{X}_{1}\right)-h\left(\mathbf{Y}_{D} \mid \mathbf{X}_{1}, \mathbf{X}_{D}\right) \\
& \stackrel{\left(b_{1}\right)}{=} E_{x_{1}}\left[h\left(\mathbf{Y}_{D} \mid \mathbf{X}_{1}=\mathbf{x}_{1}\right)-h\left(\mathbf{Z}_{D}\right)\right. \\
& =E_{x_{1}}\left[h\left(\mathbf{Y}_{D} \mid \mathbf{X}_{1}=\mathbf{x}_{1}\right)-\log \left[(\pi e)^{N_{D}} \operatorname{det}\left(I_{N_{D}}\right)\right]\right. \\
& \leq E_{x_{1}}\left[\log \left[(\pi e)^{N_{D}} \operatorname{det}\left(\operatorname{Var}\left(\mathbf{Y}_{D} \mid \mathbf{X}_{1}=\mathbf{x}_{1}\right)\right)\right]\right]- \\
& \log \left[(\pi e)^{N_{D}} \operatorname{det}\left(I_{N_{D}}\right)\right]=E_{x_{1}}\left[\log \left[\operatorname{det}\left(\operatorname{Var}\left(\mathbf{Y}_{D} \mid \mathbf{X}_{1}=\mathbf{x}_{1}\right)\right)\right]\right] \\
& =\max \log \left[\operatorname{det}\left(I_{N_{D}}+\mathbf{H}_{S D} \Sigma_{\mathbf{X}_{D} \mid \mathbf{X}_{1}=\mathbf{x}_{1}} \mathbf{H}_{S D}^{\dagger}\right)\right] \\
& \stackrel{\left(b_{2}\right)}{=} \max \log \left[\operatorname{det}\left(I_{N_{D}}+\mathbf{H}_{S D}\left(\Sigma_{D D}-\Sigma_{D 1} \Sigma_{11}^{-1} \Sigma_{D 1}^{\dagger}\right) \mathbf{H}_{S D}^{\dagger}\right)\right] \\
& \stackrel{\left(b_{3}\right)}{=} \max \log \left[\operatorname{det}\left(I_{N_{D}}+\mathbf{H}_{S D} \Sigma_{D D}^{\frac{1}{2}}\left(I-\mathbf{A A}^{\dagger}\right) \Sigma_{D D}^{\frac{1}{2}} \mathbf{H}_{S D}^{\dagger}\right)\right] \\
& \stackrel{\left(b_{4}\right)}{\leq} \log \left[\operatorname{det}\left(I_{N_{D}}+\left(1-\rho^{2}\right) \mathbf{H}_{S D} \Sigma_{D D} \mathbf{H}_{S D}^{\dagger}\right)\right] \text {. }
\end{aligned}
$$

In this derivation, step $\left(b_{1}\right)$ follows from the definition of conditional entropy. Next, to get the result in step $\left(b_{2}\right)$,

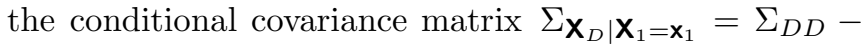
$\Sigma_{D 1} \Sigma_{11}^{-1} \Sigma_{D 1}^{\dagger}$ is considered. Further, to achieve the result in step $\left(b_{3}\right)$, we can write

$$
\begin{aligned}
& \Sigma_{\mathbf{X}_{D} \mid \mathbf{X}_{1}=\mathbf{x}_{1}}=\Sigma_{D D}-\Sigma_{D 1} \Sigma_{11}^{-1} \Sigma_{D 1}^{\dagger} \\
&=\Sigma_{D D}^{\frac{1}{2}}\left(I-\Sigma_{D D}^{\frac{-1}{2}} \Sigma_{D 1} \Sigma_{11}^{-\frac{1}{2}} \Sigma_{11}^{-\frac{1}{2}} \Sigma_{D 1}^{\dagger} \Sigma_{D D}^{-\frac{1}{2}}\right) \Sigma_{D D}^{\frac{1}{2}} \\
&=\Sigma_{D D}^{\frac{1}{2}}\left(I-\mathbf{A A}^{\dagger}\right) \Sigma_{D D}^{\frac{1}{2}}
\end{aligned}
$$

where the last step is obtained by assuming that $\mathbf{A}=$ $\Sigma_{D D}^{-\frac{1}{2}} \Sigma_{D 1} \Sigma_{11}^{-\frac{1}{2}}$. Further, $\Sigma_{D D}=E\left[\mathbf{X}_{D} \mathbf{X}_{D}^{\dagger}\right]$ and $\Sigma_{11}=$ $E\left[\mathbf{X}_{1} \mathbf{X}_{1}^{\dagger}\right]$ represent the correlation matrices. $\Sigma_{D 1}=$ $E\left[\mathbf{X}_{D} \mathbf{X}_{1}^{\dagger}\right]$ represent the covariance matrix. In addition, in this derivation, step $\left(b_{4}\right)$ is obtained by using the result from lemma $3.1[9]$ in which $\left(I-\mathbf{A A}^{\dagger}\right) \leq\left(1-\rho^{2}\right) I_{M_{s}}$ and $\rho \in[0,1]$.

To this extent, we are able to derive the achievable rate of the Gaussian MIMO-ORC in the phase of multiple access. In this phase, both the source and the relay transmit to the destination. In particular, the sum rate, $I\left(\mathbf{X}_{D}, \mathbf{X}_{1}, \mathbf{Y}_{D}\right)$, is expressed by

$$
\begin{aligned}
& C_{1}=I\left(\mathbf{X}_{D}, \mathbf{X}_{1}, \mathbf{Y}_{D}\right)=h\left(\mathbf{Y}_{D}\right)-h\left(\mathbf{Y}_{D} \mid \mathbf{X}_{D}, \mathbf{X}_{1}\right) \\
& =h\left(\mathbf{Y}_{D}\right)-h\left(\mathbf{Z}_{D}\right)=h\left(\left[\begin{array}{ll}
\mathbf{H}_{S D} & \mathbf{H}_{R D}
\end{array}\right]\left[\begin{array}{l}
\mathbf{X}_{D} \\
\mathbf{X}_{\mathbf{1}}
\end{array}\right]+\mathbf{Z}_{D}\right) \\
& -\log \left[(\pi e)^{N_{D}} \operatorname{det}\left(I_{N_{D}}\right)\right] \stackrel{\left(d_{1}\right)}{\leq} \\
& \log \left[(\pi e)^{N_{D}} \operatorname{det}\left(\operatorname{Cov}\left(\left[\begin{array}{ll}
\mathbf{H}_{S D} & \mathbf{H}_{R D}
\end{array}\right]\left[\begin{array}{l}
\mathbf{X}_{D} \\
\mathbf{X}_{1}
\end{array}\right]+\mathbf{Z}_{D}\right)\right)\right] \\
& -\log \left[(\pi e)^{N_{D}} \operatorname{det}\left(I_{N_{D}}\right)\right]=
\end{aligned}
$$

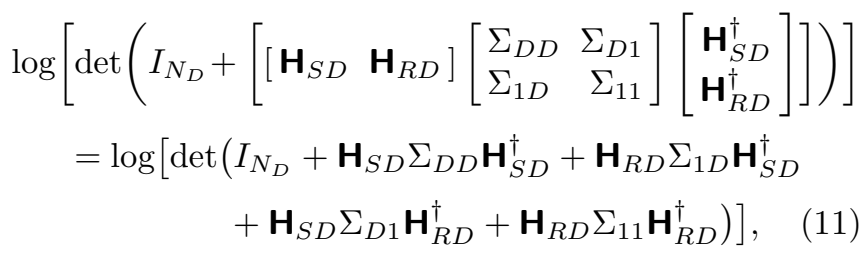

where step $\left(d_{1}\right)$ is obtained since circularly symmetric complex Gaussian distribution maximizes the entropy [6].

Finally, the rates in (5), (6) and (8) forms that shown in Theorem 1.

To this end, we are eager to introduce an algorithm to compute the rates in Theorem 1. The main goal of this algorithm is to perfectly allocate the available power at source such that the broadcast term, $\left(C_{2}+C_{3}\right)$, and the multiple access term, $\left(C_{1}\right)$, are nearly equal.

- Use the water-filling technique and antenna selection to compute $C_{1}, C_{2}$, and $C_{3}$.

- In the case of $C_{1}-\left(C_{2}+C_{3}\right)>\delta$, where $\delta$ is a pre-defined threshold, then, allocate more power to transmit $\mathbf{X}_{R}$ until $C_{1}-\left(C_{2}+C_{3}\right) \leq \delta$.

- In the case of $\left(C_{2}+C_{3}\right)-C_{1}>\delta$, then, allocate more power to transmit $\mathbf{X}_{D}$ until $\left(C_{2}+C_{3}\right)-C_{1} \leq \delta$.

From this algorithm, it is clear that power allocation is mainly performed at the source to determine at first the value of $\alpha$ such that $\alpha P_{S}$ is used to directly transmit to destination and $\bar{\alpha} P_{S}$ to transmit to relay. Thereafter, water-filling in conjunction with antenna selection are used to determine which antennas to use for transmission. Even thought that the authors in [11] showed that employing either water-filling technique or antenna selection can increase the capacity of a given system with multiple antennas, Telatar [6] showed that water-filling can be used to achieve the capacity of the Gaussian MIMO channel.

Next, many numerical results are shown to validate our theoretical results. In this regard, as previously mentioned, the capacity of the MIMO-ORC is equal to the max-flow min-cut upper bound of the general MIMO relay channel [2]. 


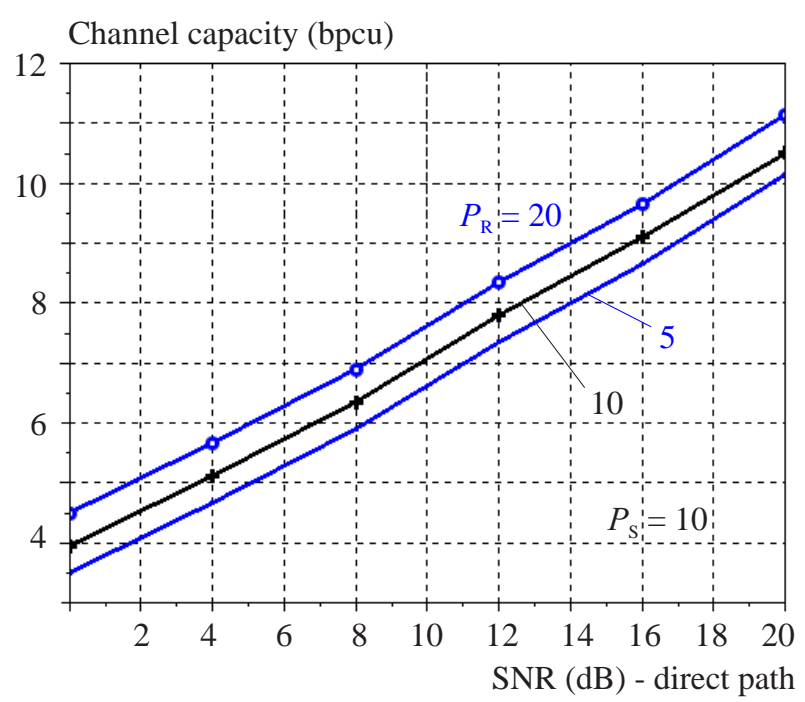

Fig. 2. Achievable Capacity of the Gaussian MIMO-ORC versus the signal-to-noise ratio of the direct path for different values of average power at the transmitting nodes

\section{Numerical analysis}

We now ready for presenting some numerical examples that show the importance employing multiple transmit and multiple receive antennas, and also transmission over different frequency bands. In these simulations, we follow the guidelines that were presented in Algorithm. Unless otherwise indicated, the average power at each source is set to 10 , ie, $P_{S}=P_{R}=10$. The noise variance at all receive nodes is normalized to 1 . In addition, the number of antennas at all nodes is set to 2 . Further, the channel gains are randomly selected based on Gaussian distribution. Finally, the results are averaged over 200 thousands iterations.

Fig. 2 shows the relation between the channel capacity, in bit per channel use (bpcu), versus the signal-to-noise ratio of the direct path for different values of average power at the transmitting nodes. In particular, this figure indicates that as the average power per transmit node increases so does the achievable capacity.

The relation between the achievable capacity and the number of transmit antennas at the source, for different values of receive antennas at both the relay and the destination, is also shown in Fig. 3. This figure clearly indicates that as the number of transmit antennas at the source increases so does the achievable capacity. Further, the achievable capacity is significantly increased by exploiting large number of receive antennas at both the relay and the destination. For instance, the number of receive antennas at the destination are increased from 2 antennas to 8 antennas in a case. Further, in another case, 8 receive antennas are utilized at both the relay and the destination. This means that as long as the number of transmit and receive antennas increases so does the achievable rate.

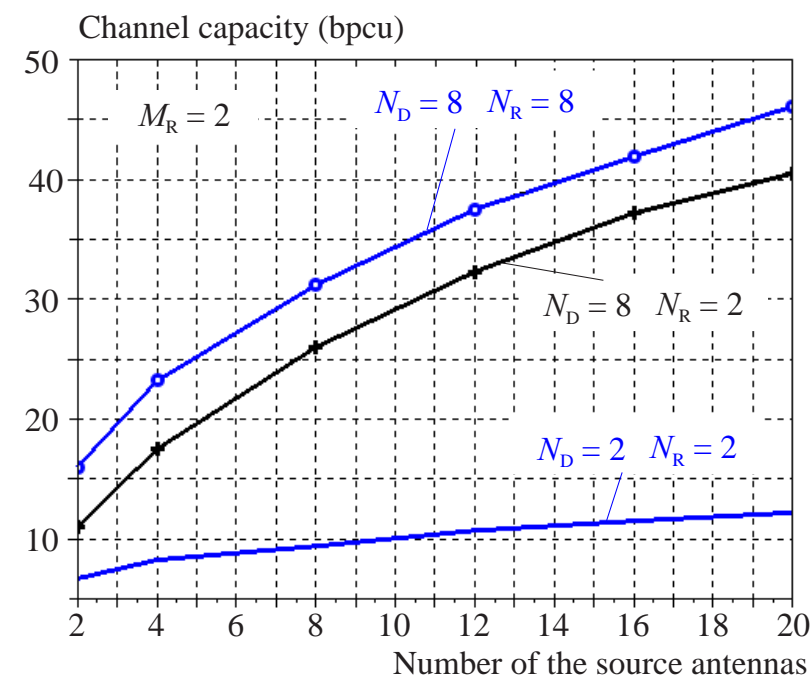

Fig. 3. Achievable capacity of the Gaussian MIMO-ORC versus the number of transmit antennas at the source for different values of receive antennas at both the relay and the destination

\section{Conclusion}

In this paper, the transmission over MIMO-ORC has been investigated. In particular, a closed-form expression for the capacity of the MIMO-ORC has been derived. Additionally, we note that this capacity is equal to the max-flow min-cut upper bound of the general MIMO relay channel [sinz2005]. Then, not only to this extent, but rather an iterative transmission algorithm has been proposed to achieve the capacity. Finally, many numerical examples have presented to show the value of employing different frequency bands and also the value of utilizing large number of transmit and receive antennas.

\section{REFERENCES}

[1] T. Cover and A. Gamal, "Capacity Theorems for the Relay Channel, IEEE Trans. Inf. Theory, vol. 25, pp. 572-584, Sep 1979

[2] A. El-Gamal and S. Zahedi, "Capacity of a Class of Relay Channels with Orthogonal Components", IEEE Trans. Inf. Theory, vol. 51, pp. 1815-1817, May 2005.

[3] Z. Al-qudah, "Achievable Rates of Gaussian Two-Way Relay Channel with Orthogonal Components and Partial Decoding at Relay", Electronics Lett., vol. 52, no. 3, pp. 200-202, 2016.

[4] O. Sahin, O. Simeone, and E. Erkip, "Interference Channel with an Out-of-Band Relay", IEEE Trans. Inf. Theory, vol. 57, pp. 2746-2764, May 2011.

[5] Z. Al-qudah, "Achievable Rates of a State-Dependent Relay Channel with Orthogonal Components", IET Commun., vol. 10, no. 16, pp. 2149-2153, 2016.

[6] I. E. Telatar, "Capacity of Multi-Antenna Gaussian Channels", Eur. Trans. Telecommun., vol. 10, pp. 585-596, 1999.

[7] T. Cover and J. Thomas, "Elements of Information Theory", John Wiley \& Sons, 2006.

[8] G. Caire and S. Shamai, "On the Achievable Throughput of a Multiantenna Gaussian Broadcast Channel", IEEE Trans. Inf. Theory, vol. 49, no. 7, pp. 1691-1706, 2003. 
[9] B. Wang, J. Zhang, and A. Host-Madsen, "On the Capacity of MIMO Relay Channels", IEEE Trans. Inf. Theory, vol. 51, pp. 29-43, Jan 2005.

[10] C.-K. Wen, K.-K. Wong, and C. Ng, "On the Asymptotic Properties of Amplify-and-Forward MIMO Relay Channels", IEEE Trans. Commun., vol. 59, pp. 590-602, Feb 2011.

[11] J. V. Cuan-Cortes, C. Vargas-Rosales, and D. Munoz-Rodriguez, "MIMO Channel Capacity using Antenna Selection and Water Pouring", EURASIP J. Wireless Commun. and Networking, vol. 2014, p. 228, Dec 2014.

[12] S. Simoens, O. Munoz-Medina, J. Vidal, and A. del Coso, "On the Gaussian MIMO Relay Channel with Full Channel State Information", IEEE Trans. Signal Process., vol. 57, pp. 3588-3599, Sep 2009.

[13] X. Hassan and N. Fernando, "Massive MIMO Wireless Networks: An Overview", Electronics, 2017.
Received 26 October 2019

Zouhair Al-qudah received the BSc, $\mathrm{MSc}$, and $\mathrm{PhD}$ all in Electrical (Wireless Communication) Engineering from Yarmouk University, Jordan, in 2002, Kalmar University College, Sweden in 2006, and Southern Methodist University at Dallas, Texas in 2013, respectively. Since August 2013, he has been with Al-Hussein Bin Talal University at Ma'an, Jordan, where he is currently an Associate Professor. His research interest includes coding and information theory in wireless communicatio nsystems, cooperative communications $5 \mathrm{G}$ communication, resource allocation problems, interference management and signal processing. 